\section{Stereotactic \\ and Functional \\ Neurosurgery}

\title{
Quality Assurance in Stereotactic Radiosurgery/Radiotherapy according to DIN 6875-1
}

\author{
A. Mack ${ }^{a, e}$ G. Mack ${ }^{d, e}$ S. Scheib ${ }^{i}$ H. Czempiel ${ }^{f} \quad$ H.J. Kreiner ${ }^{f}$ N.J. Lomax ${ }^{i}$ \\ S. Gianolini' M. Rieker \\ D. Weltz \\ R. Wolff ${ }^{a}$ \\ A. Muacevic ${ }^{g}$ \\ B. Wowra ${ }^{\mathrm{h}}$ \\ H.D. Böttcher ${ }^{b}$ V. Seifert ${ }^{\mathrm{c}}$ \\ ${ }^{a}$ Gamma Knife Centre Frankfurt, GKF GmbH, ${ }^{b}$ Department of Radiotherapy, and ${ }^{\mathrm{c}}$ Department of Neurosurgery, \\ University Hospital Frankfurt, Frankfurt, ${ }^{\mathrm{d}}$ Department of Experimental Physics, University of Tübingen, and \\ ePTGR GmbH, Tübingen, ${ }^{f}$ GKS GmbH, ${ }^{9}$ Department of Neurosurgery, University Hospital Munich,

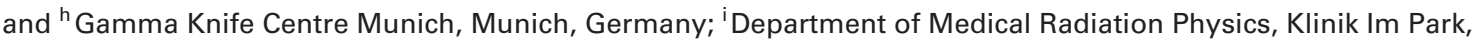 \\ Zurich, Switzerland
}

\section{Key Words}

Quality assurance $\cdot$ Stereotactic radiosurgery $\cdot$

Sterotactic radiotherapy · System test · DIN 6875-1

\begin{abstract}
The new DIN ('Deutsche Industrie-Norm') 6875-1, which is currently being finalised, deals with quality assurance (QA) criteria and tests methods for linear accelerator and Gamma Knife stereotactic radiosurgery/radiotherapy including treatment planning, stereotactic frame and stereotactic imaging and a system test to check the whole chain of uncertainties. Our existing QA program, based on dedicated phantoms and test procedures, has been refined to fulfill the demands of this new DIN. The radiological and mechanical isocentre corresponded within $0.2 \mathrm{~mm}$ and the measured $50 \%$ isodose lines were in agreement with the calculated ones within less than $0.5 \mathrm{~mm}$. The measured absorbed dose was within $3 \%$. The resultant output factors measured for the 14-, 8- and 4-mm collimator helmet were $0.9870 \pm 0.0086,0.9578$ \pm 0.0057 and $0.8741 \pm 0.0202$, respectively. For 170 con-
\end{abstract}

secutive tests, the mean geometrical accuracy was 0.48 $\pm 0.23 \mathrm{~mm}$. Besides QA phantoms and analysis software developed in-house, the use of commercially available tools facilitated the QA according to the DIN 6875-1 with which our results complied.

Copyright $(2004$ S. Karger AG, Basel

\section{Introduction}

Intracranial stereotactic radiosurgery (SRS) and stereotactic radiotherapy (SRT) represent a noninvasive, single (SRT) or fractionated (SRS) irradiation method for lesions within the skull, relying on the accurate localization of anatomical structures, small radiation fields (diameters down to $4 \mathrm{~mm}$ ) and steep dose gradients (up to $40 \%$ per $\mathrm{mm}$ ). To date, more than 230,000 patients, suffering from meningiomas, cerebellopontine angle schwannomas, arteriovenous malformations, pituitary adenomas, brain metastases, glial tumours or trigeminal neuralgia have been treated by $179 \mathrm{Gamma} \mathrm{Knife}{ }^{\circledR}$ centres worldwide. Quality assurance (QA) in SRS and SRT

\section{KARGER}

Fax +4161306 1234 E-Mail karger@karger.ch www.karger.com
Dr. rer. nat. Andreas Mack

Gamma Knife Zentrum/GKF GmbH

Schleusenweg 2-16, Nebengebäude 95

DE-60528 Frankfurt (Germany)

Tel. +4969677359 14, Fax +4969677359 11, E-Mail a.mack@gkfrankfurt.de 
is of utmost importance and one of the tasks of medical physicists in charge of a clinical SRS/SRT program. In the literature, quite a number of contributions addressing this issue can be found [1-15]. Recently, the draft of the new E-DIN ('Deutsche Industrie-Norm' valid in Europe) 6875-1 has been published [16] and is currently being finalised. This first part of the DIN deals with acceptance procedures and will be the basis for the second part describing regular constancy checks. Our aim was to adapt the existing QA program for the Gamma Knife to the new standard, estimate on the expenditure necessary and to give notice of this new standard.

\section{Materials and Methods}

\section{DIN 6875-1 - Introduction}

This DIN covers functional performance characteristics for stereotactically guided, percutaneous photon irradiations in the head and neck which are characterized by a high geometrical accuracy of the irradiation $(0.5-2.0 \mathrm{~mm})$ and a steep dose gradient. Test conditions and test procedures are defined including the irradiation device (adapted linear electron accelerator with fixed tertiary or micro-multileaf collimator and Gamma Knife), the treatment planning system, the stereotactic instrument and diagnostic imaging modalities [CT, MRI, angiography, digital subtraction angiography (DSA)].

The small dimensions of the therapeutically used irradiation fields require dedicated dosimetrical test procedures. An essential aim of this standard is the comparability of functional performance characteristics for different irradiation devices of different manufacturers. Additionally, test procedures and test conditions for the system test [1], simulating the complete treatment chain, are described.

The aim of this standard is also to describe acceptance and regular constancy tests of an SRS/SRT system.

\section{DIN 6875-1 - Demands}

Dosimeter. In addition to standard requirements, the geometrical resolution must be small compared to the radiation fields. In general, it is recommended to perform comparative measurements using different dosimeters such as radiochromic films, diamond detectors, thermoluminescence dosimeters and alanine pellets.

Irradiation. Irradiations in order to measure the dose distribution have to be performed in the same way as for patients including diagnostic imaging modalities (CT, MRI, angiography, DSA).

Phantom. A homogeneous, regular phantom (sphere) which allows the position of dosimeters in the central part must be used.

\section{DIN 6875-1 - Dosimetric Functional Performance}

Characteristics for Gamma Knife

There are four collimator helmets $(4,8,14$ and $18 \mathrm{~mm})$; these are geometrical projections of the collimator aperture at the unit centre point of the Gamma Knife. The superposition of all 201 beams then produces approximately spherically shaped dose distributions, with full width at half maximum dimensions (in $\mathrm{x}$ and $\mathrm{y}$ directions) of $6,11,19$ and $24 \mathrm{~mm}$, respectively, at the isocentre [2].

Output Factor. The output factor has to be determined for the 4-, 8- and 14-mm collimator helmet relative to the 18-mm colliPmator helmet, which produces the largest field size using a spherical phantom [3, 4].

Dose Distribution. For all collimator helmets, the isodose curves in three orthogonal planes, determined by the stereotactic coordinate system and their deviation to the calculated ones, have to be documented.

\section{DIN 6875-1 - Dosimetric Functional Performance}

\section{Characteristics for Linear Accelerator}

Output Factor. The output factor has to be determined on the central axis at the reference depth relative to the calibration condition for the dose monitor. Output factors have to be determined for all fixed tertiary collimators and for the minimal and maximal, as well as for a medium, rectangular field size for micro-multileaf collimators [5].

Tissue-Phantom Ratio. The tissue-phantom ratios for the same set-up as for the output factor measurements have to be measured on the central axis. For multileaf collimators, spot checks have to be performed for any field shape in order to compare the measured depth dose to the calculated one.

Dose Profiles. Normalised dose profiles with a sufficient geometrical resolution have to be documented for the same set-up as for output factor measurements at isocentre position.

\section{DIN 6875-1 - Geometrical Functional Performance}

Characteristics

Stereotactic Localisation. The relationship between image coordinates and stereotactic coordinates has to be derived with the help of fiducial systems. If non-linear image distortions apply, they have to be determined with dedicated phantoms and the patient images have to be corrected.

Target Points/Volumes in Stereotactic Space. Target coordinates are given in the stereotactic space and usually show some uncertainties due to the imaging (distortions, artefacts), stereotactic frame deformations/instabilities, fiducial determination and coordinate transformation. These geometrical uncertainties have to be estimated by using known target points in the stereotactic space and have to be determined in the same way as target coordinates for patients.

\section{DIN 6875-1 - Device Functional Performance Characteristics} for Linear Accelerator

Ideally gantry and patient table rotation axis should intersect in a single point. The following functional performance characteristics are specific for the given device and have to be determined [6].

(1) Distance (in millimetres) of gantry rotation axis and isocentric patient table rotation axis (geometric isocentre).

(2) Radius (in millimetres) of the isocentre sphere, which is the smallest sphere through which the central ray of the radiation beam runs for all gantry and table rotation angles (Winston-Lutz test, radiation isocentre).

(3) The deviation of the geometrical and radiation isocentre from the optical indication of the isocentre must be specified (in millimetres) in three orthogonal planes within the room coordinate system. 
Table 1. Measured output factors (OF) using different dosimeters

\begin{tabular}{llcc}
\hline Dosimeter & $14 \mathrm{~mm} \mathrm{OF} \pm 1 \mathrm{SD}$ & $8 \mathrm{~mm}$ OF $\pm 1 \mathrm{SD}$ & $4 \mathrm{~mm}$ OF $\pm 1 \mathrm{SD}$ \\
\hline Pin point chamber & $0.9815 \pm 0.0009$ & $(0.9280 \pm 0.0020)$ & $(0.6630 \pm 0.0060)$ \\
Liquid ionisation chamber & $0.9829 \pm 0.0007$ & $0.9523 \pm 0.0007$ & $0.8930 \pm 0.0005$ \\
Diode & $0.9834 \pm 0.0011$ & $0.9550 \pm 0.0020$ & $0.8874 \pm 0.0072$ \\
Diamond detector & $0.9854 \pm 0.0004$ & $0.9569 \pm 0.0004$ & $0.8797 \pm 0.0018$ \\
LIF micro-cubes & $0.9839 \pm 0.0015$ & $0.9561 \pm 0.0027$ & $0.8826 \pm 0.0071$ \\
TLD rods & $0.9842 \pm 0.0010$ & $0.9558 \pm 0.0028$ & $0.8821 \pm 0.0068$ \\
Alanine dosimeter & $0.9870 \pm 0.0130$ & $0.9590 \pm 0.0120$ & $0.8350 \pm 0.0180$ \\
GAFCHROMIC film & $1.0080 \pm 0.0150$ & $0.9700 \pm 0.0060$ & $0.8592 \pm 0.0147$ \\
Mean value & $0.9870 \pm 0.0086^{1}$ & $0.9578 \pm 0.0057^{1}$ & $0.8741 \pm 0.0202^{1}$ \\
\hline
\end{tabular}

The figures in parentheses for the pin point chamber were not taken into account for calculating the mean values due to systematic errors [5].

${ }^{1}$ Standard deviation of mean value.

\section{DIN 6875-1 - Device Functional Performance Characteristics} for Gamma Knife

The deviation of the geometrical centre to the isocentre (unit centre point) must be specified (in millimetres) for three orthogonal planes within the room coordinate system.

\section{DIN 6875-1 - Treatment Planning System}

The aim of these tests is to document the agreement between dose, dose distribution and geometrical localisation of the calculated and measured parameters [7].

(1) Data transfer from diagnostic imaging modalities to the treatment planning system including coordinate transformation and determination of known target points in stereotactic coordinates. The distances between known and detected target points (in millimetres) have to be determined in three orthogonal planes.

(2) The maximal deviations and full width at half maximum values of calculated and measured isodose curves for at least three representative dose distributions have to be determined (in millimetres).

(3) The treatment planning system has to make available all necessary dosimetric data (e.g. $\mathrm{D}_{\max }, \mathrm{D}_{\min }, \mathrm{D}_{50}$, dose volume histogram) to document the stereotactic irradiation.

\section{DIN 6875-1 - Imaging Accuracy}

In general, distortions in diagnostic imaging modalities are an essential contribution to the total geometrical accuracy. Therefore, the imaging modalities used must be checked and prior to starting patient treatments the geometrical uncertainty of the imaging modalities must be known. This uncertainty (mean and max in millimetres) must be checked routinely, especially after service and repair interruptions [8-10].

In order to check this, dedicated phantoms mounted to the stereotactic frame and imaged with the same imaging protocols as for patients must be used. The marker positions within the phantom must be known within an uncertainty of $<0.2 \mathrm{~mm}$.

DIN 6875-1 - System Test

This test quantifies the overall accuracy of any stereotactic treatment (SRS/SRT) by simulating a single isocentre irradiation (fig. 7). In addition to other imaging modalities, the MR images are an integral part of the stereotactic system (SRS/SRT) and therefore must be tested. In order to evaluate how precisely a target can be irradiated, a marked GAFCHROMIC ${ }^{\circledR}$ film is embedded between two PMMA plates both containing a capillary cross which can be filled with copper sulphate and coincides with the marked centre of the film. The film has additional marks at the edge so that different scans of the film can be positioned automatically. The capillary cross or film plane together with the head phantom is adjusted either axially or sagittally within the stereotactic frame. The position of the target is kept unknown until the dose is applied. After the MR/CT images have been imported, stereotactically defined and the dose planning has been performed, the phantom is irradiated according to the data in the planning protocol. Finally, the film coloured by the radiation is evaluated. The deviation from the centre of gravity of the coloured area to the central point marked at the beginning is measured $[1,11]$.

\section{DIN 6875-1 - Verification of Calculated Dose Calculations}

Calculated dose distributions can be verified by simulating the application of dose using the head phantom. After adjusting the coordinates and applying the dose, a stack of embedded films is read and analysed [12-15]. The resulting measured dose distributions can be compared to the calculated ones.

\section{Results}

\section{Dosimetric Functional Performance Characterisitics for Gamma Knife}

The output factors for the 4-, 8- and 14-mm collimator helmet relative to the $18-\mathrm{mm}$ collimator helmet have been measured (table 1) with different detectors which in principle fulfill the requirements for narrow photon beam dosimetry $[3,4]$. The detectors were placed with an uncertainty of approximately $0.1 \mathrm{~mm}$ in the centre of a homo- 
Fig. 1. Calculated (broken lines) and measured (diamond detector, dots) relative Gamma Knife dose profiles along the y-axis for the 4-, 8-, 14- and 18-mm collimator helmet.

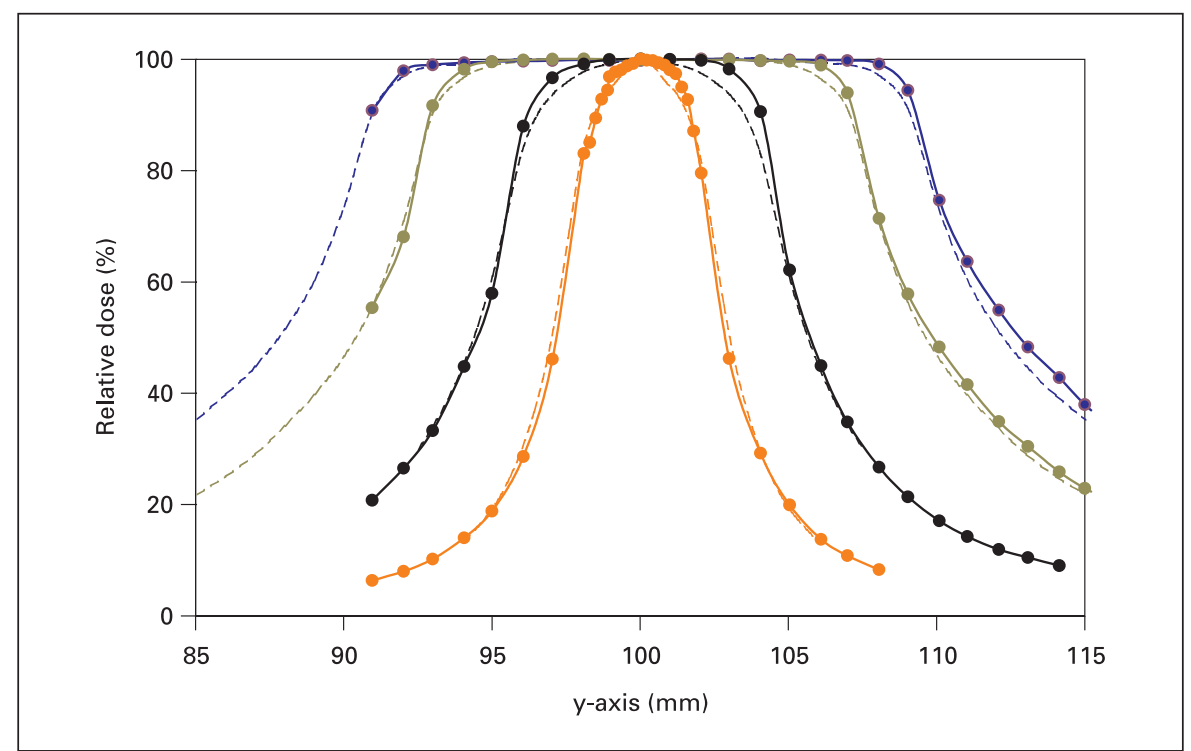

geneous polystyrene sphere $($ diameter $=16 \mathrm{~cm})$ which was mounted in the mechanical centre of the Gamma Knife.

For all collimator helmets, dose profiles with different detectors and radiochromic films have been measured (fig. 1) using the spherical head phantom. Similar results have been obtained comparing measured with calculated isodose curves in orthogonal planes using GAFCHROMIC films.

\section{Imaging Accuracy and Geometrical Functional \\ Performance Characteristics}

In order to check distortions in CT and MR images, a cylindrical phantom consisting of 145 rods, each $10 \mathrm{~mm}$ apart, was mounted in the stereotactic frame in such a way that the central rod coincided with a defined stereotactic coordinate $(\mathrm{x}=100 \mathrm{~mm}, \mathrm{y}=100 \mathrm{~mm})$ in axial planes [9]. The phantom was imaged with the same imaging protocols used for patients (fig. 2a, b).

The DICOM images are then postprocessed by a software module (TOPAS) calculating the displacement vectors for each rod, assuming a perfect geometrical match in the central position (fig. 3). Using stereotactic CT images, the mean radial deviation was found to be $0.1 \pm$ $0.06 \mathrm{~mm}$ with a maximum deviation of $0.6 \mathrm{~mm}$ which is nearly independent of the distance from the central reference rod. However, in MR images, the mean radial deviation was up to $0.7 \pm 0.3 \mathrm{~mm}$ with a maximum of $1.3-1.6 \mathrm{~mm}$, depending on the imaging protocol used. Systematically increasing deviations were found with increasing distance from the central reference rod.
By using the 'known target phantom' (fig. 4) with embedded targets at known geometrical positions, either 45 steel spheres for CT or 21 cross hairs for MR (each consisting of 5 glass vials) following results could be obtained (table 2).

With a similar phantom (fig. 5), image distortions in DSA images have been estimated to be up to $3.5 \mathrm{~mm}$ in the peripheral image regions due to the curved input phosphor of the image intensifier (fig. 6).

\section{Mechanical Isocentre (Unit Centre Point) Stability}

The measured deviation of the radiological centre to the mechanical centre (unit centre point) of the Gamma Knife was measured in three orthogonal planes with a special tool representing the mechanical centre by a pin point marking a GAFCHROMIC film. The deviation found for xy measurements was $0.20 \pm 0.13 \mathrm{~mm}$.

\section{System Test}

The system test was performed using a spherical, tissue-equivalent phantom with an MR visible cross at an unknown target position, to which a GAFCHROMIC film was placed (fig. 7). After mounting this phantom into the stereotactic frame, stereotactic MR images were taken. The shot position for a 4-mm collimator helmet was planned to coincide with the visible crossing point. By densitometric analyses of the film after the irradiation, the deviation of the planned to the irradiated shot position is determined. 

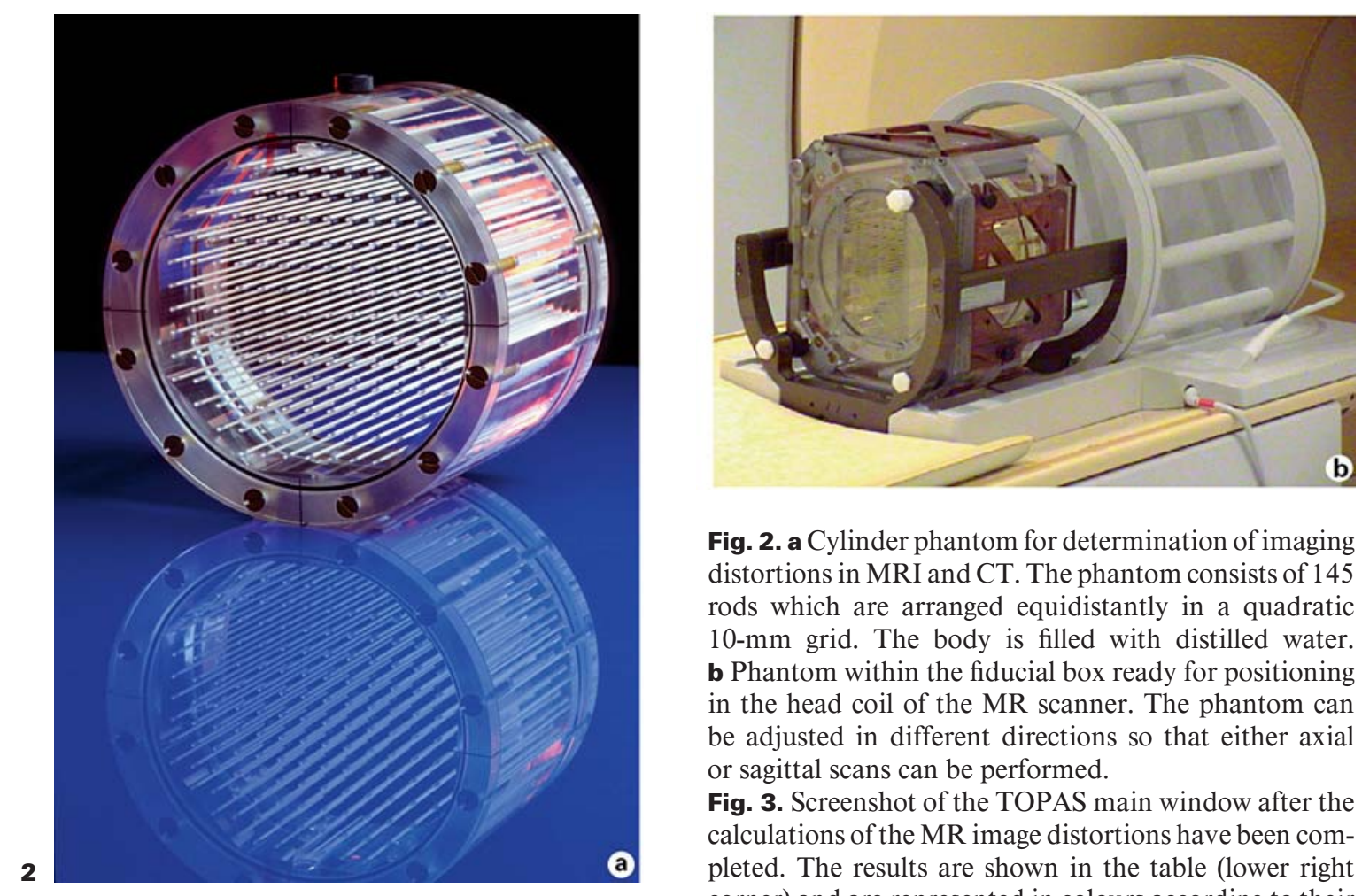

Fig. 2. a Cylinder phantom for determination of imaging distortions in MRI and CT. The phantom consists of 145 rods which are arranged equidistantly in a quadratic $10-\mathrm{mm}$ grid. The body is filled with distilled water. b Phantom within the fiducial box ready for positioning in the head coil of the MR scanner. The phantom can be adjusted in different directions so that either axial or sagittal scans can be performed.

Fig. 3. Screenshot of the TOPAS main window after the calculations of the MR image distortions have been completed. The results are shown in the table (lower right corner) and are represented in colours according to their magnitude.

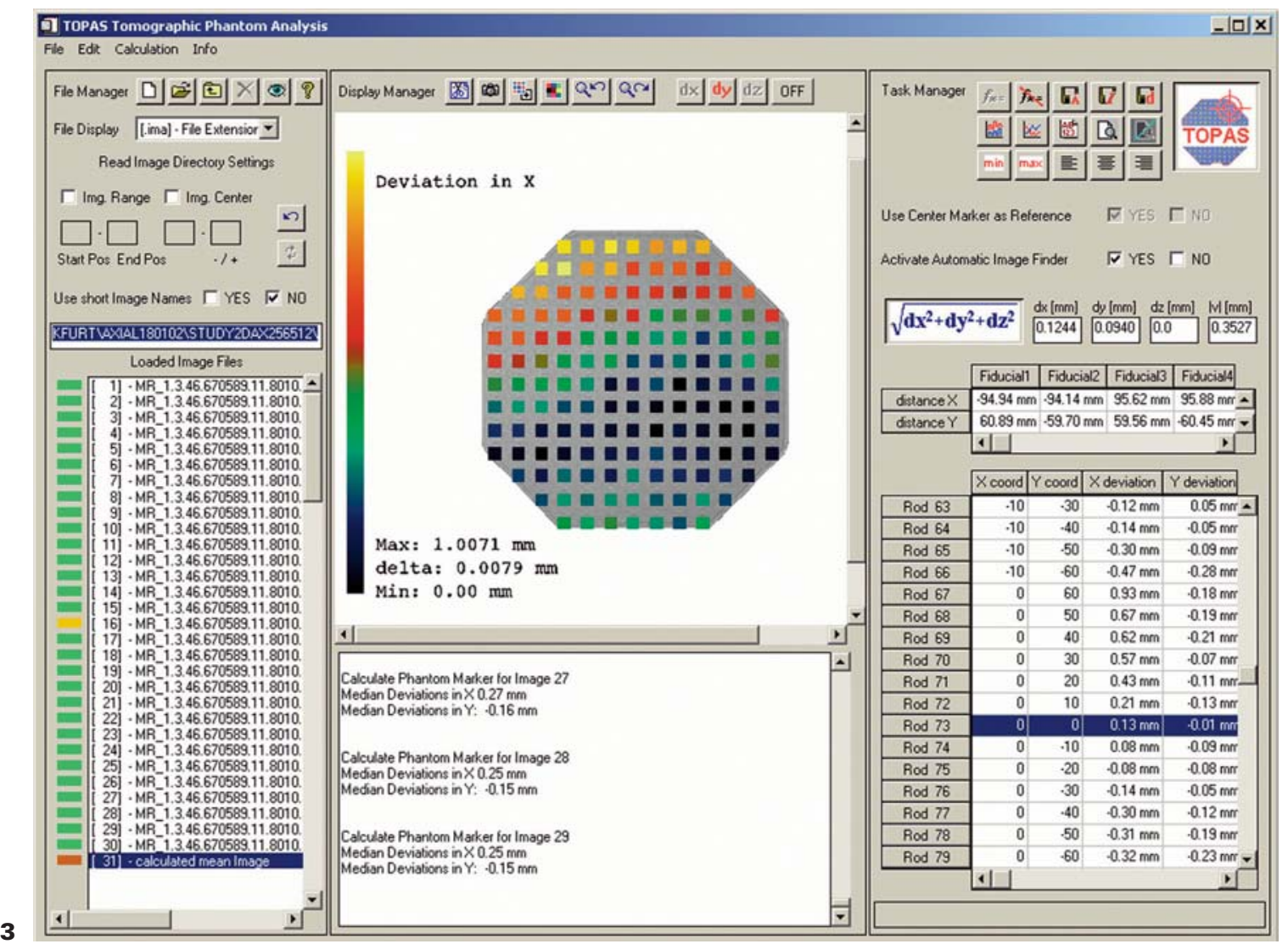



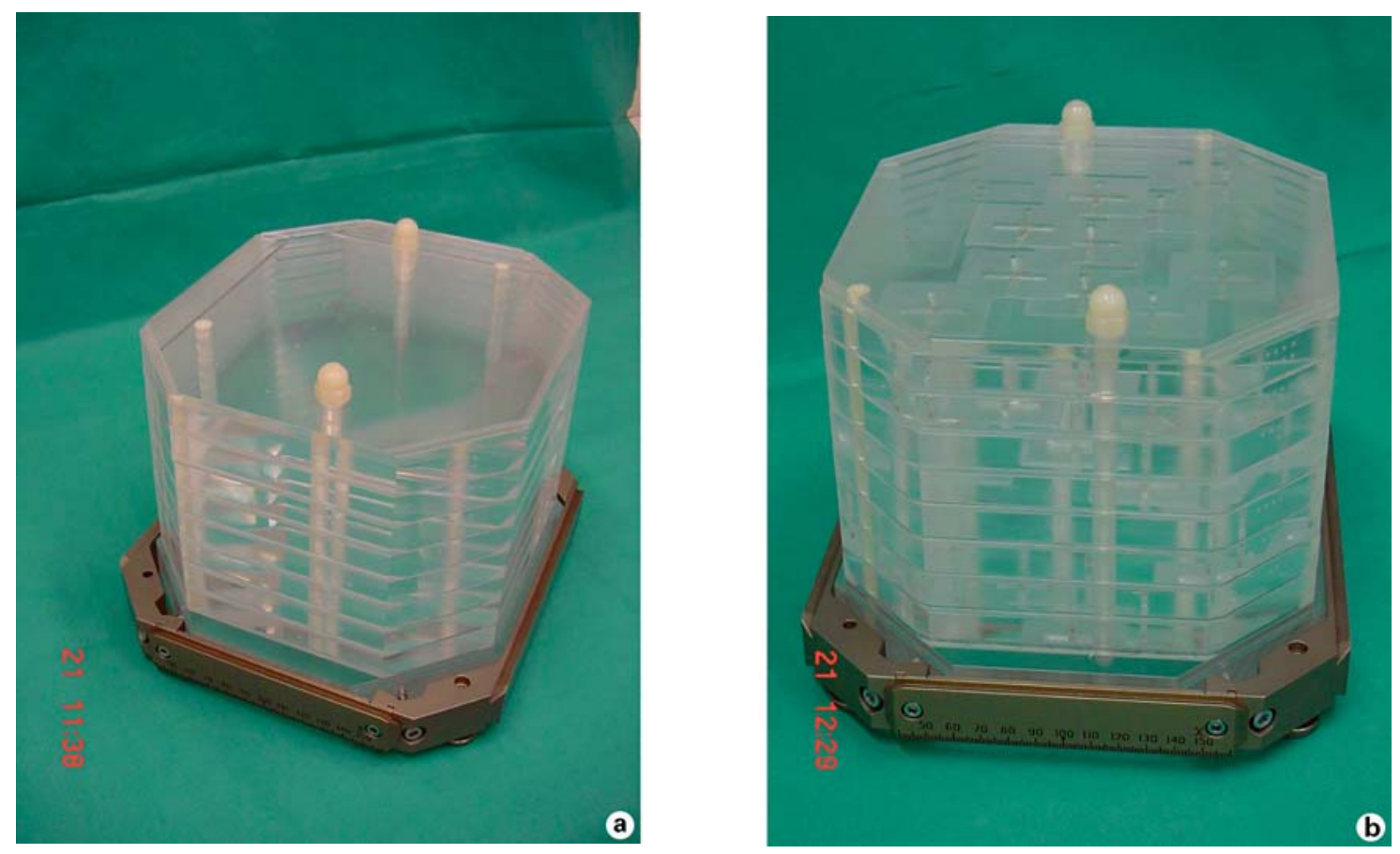

Fig. 4. Known target phantom for stereotactic imaging. a Slab phantom with 45 embedded steel spheres ( $\varnothing 1 \mathrm{~mm}$ ) for QA when using CT, angiography and DSA. b Slab phantom with 21 embedded cross hairs (each cross hair consists of 5 glass vials with outer $\varnothing 3 \mathrm{~mm}$ and inner $\varnothing 2 \mathrm{~mm}$ ) for verification of geometrical localisation in MRI.
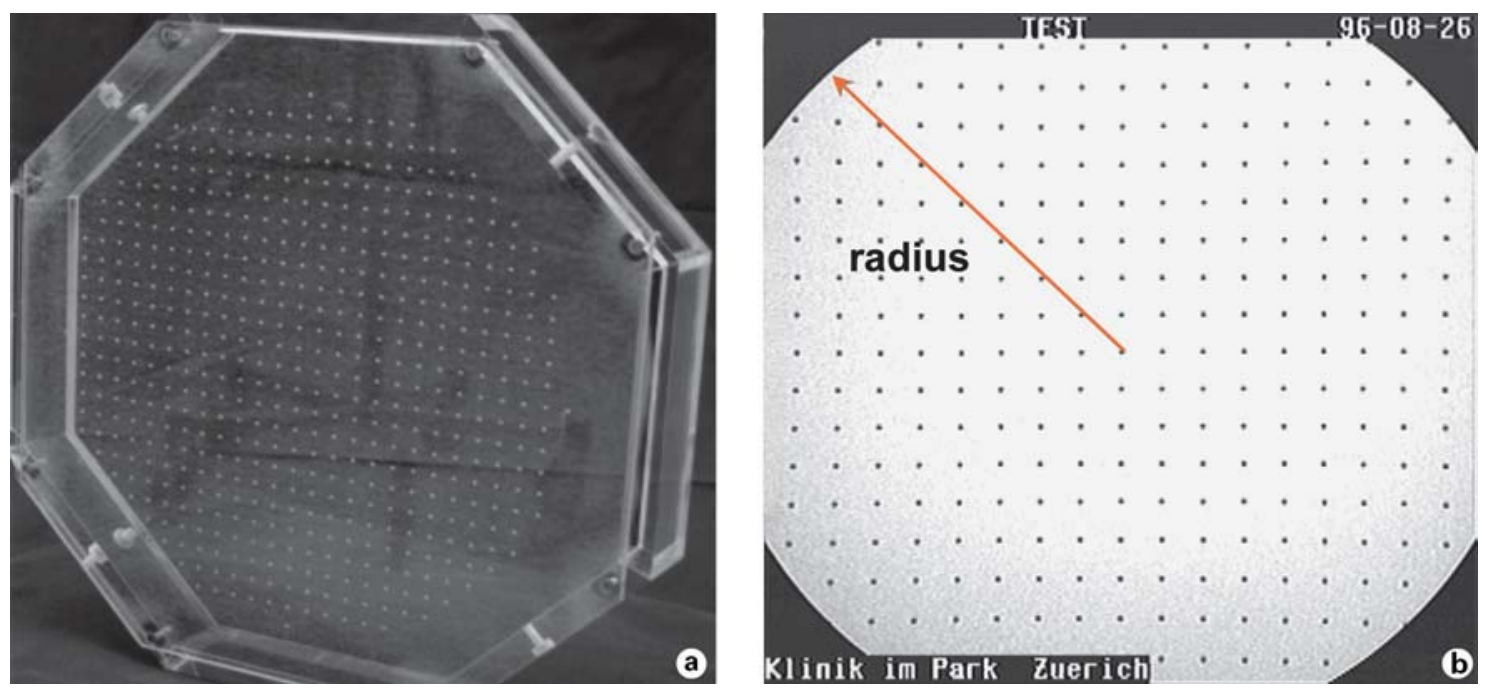

Fig. 5. Phantom for stereotactic DSA. a Three-slab phantom with embedded steel sheres for QA of DSA. b Representation on the monitor showing the usual distortions ('cushing effect').

The results (table 3) evaluating 170 system tests within 5 years show that the mean displacement vector of the complete system is $0.48 \pm 0.23 \mathrm{~mm}$ (mean $\pm \mathrm{SD}$ ). Factors having a significant influence on the overall accuracy are associated with MRI parameters. Test results based on axial images (xy plane; $0.42 \pm 0.24 \mathrm{~mm}$ ) are significantly superior to coronal images ( $\mathrm{xz}$ plane; $\mathrm{x}=0.60 \pm$ $0.02 \mathrm{~mm}$ ). Furthermore, the 3 -dimensional mpr sequence $(0.40 \pm 0.19 \mathrm{~mm})$ is significantly superior to the $T_{1}$ weighted SE sequences $(0.66 \pm 0.24 \mathrm{~mm})$ [1]. 


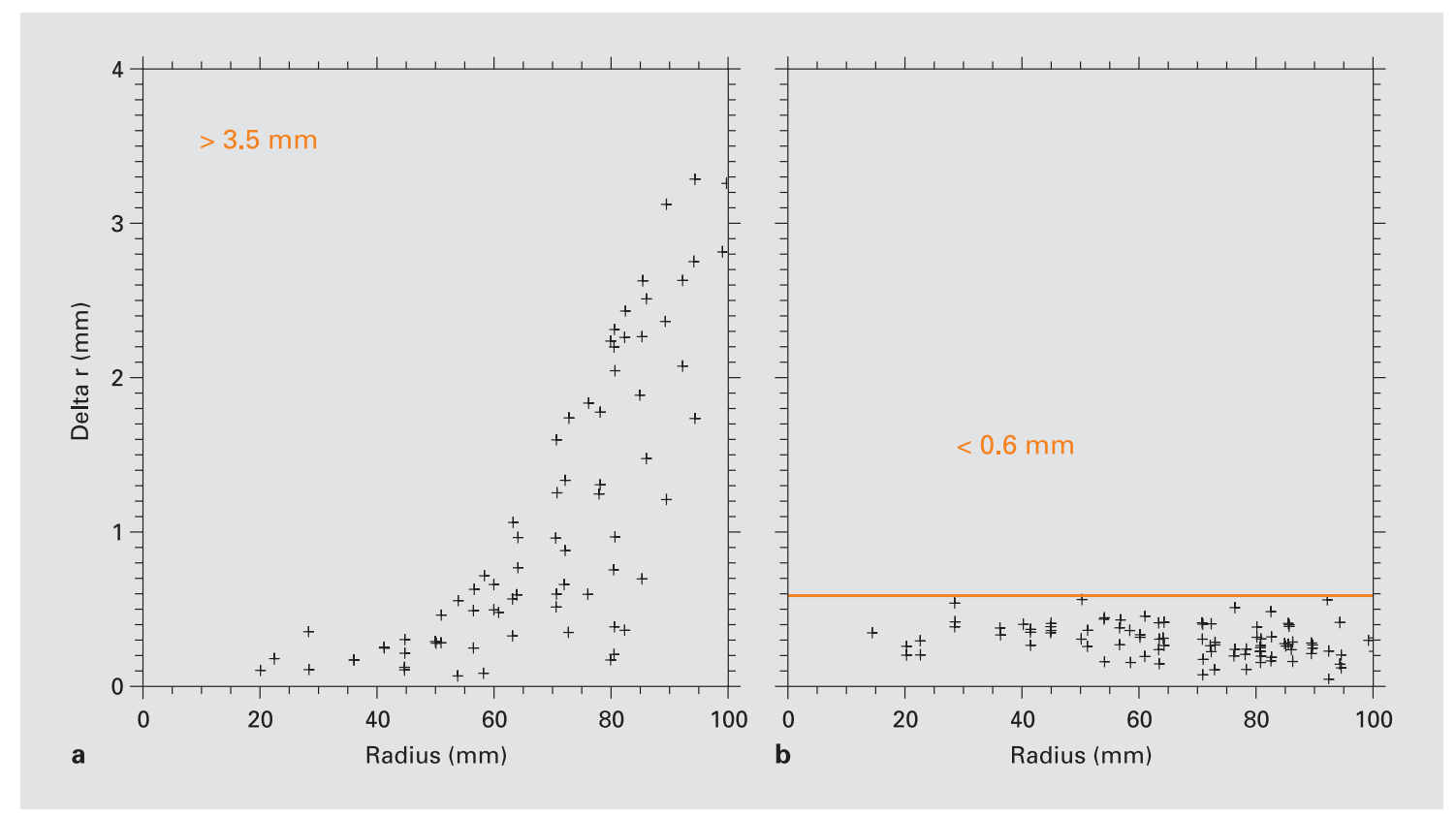

Fig. 6. Results of QA measurements for a DSA (Philips Integris Allura) using the 3-slab phantom. a The deviations of the spheres to the real given positions as a function of distance to the centre. $\mathbf{b}$ After applying a correction algorithm, all markers appear within $0.6 \mathrm{~mm}$ to the real given position in the phantom.

Table 2. Results (mm) from verifying 21 geometrically known targets in stereotactic space

\begin{tabular}{|c|c|c|c|c|c|c|c|c|c|c|c|}
\hline \multirow{2}{*}{$\begin{array}{l}\text { Marker } \\
\text { No. }\end{array}$} & \multicolumn{3}{|c|}{ Target coordinates } & \multicolumn{3}{|c|}{ Measured coordinates } & \multicolumn{4}{|c|}{ Absolute errors } & \multirow[t]{2}{*}{ Distance } \\
\hline & $\mathrm{x}$ & $\mathrm{y}$ & $\mathrm{z}$ & $\mathrm{x}$ & $\mathrm{y}$ & $\mathrm{Z}$ & $\mathrm{dx}$ & dy & $\mathrm{dz}$ & $\mathrm{dr}$ & \\
\hline 1 & 100.0 & 70.0 & 45.0 & 100.1 & 70.1 & 45.2 & 0.1 & 0.1 & 0.2 & 0.24 & 62.6 \\
\hline 2 & 75.0 & 100.0 & 45.0 & 75.2 & 100.2 & 45.2 & 0.2 & 0.2 & 0.2 & 0.35 & 60.4 \\
\hline 3 & 150.0 & 40.0 & 60.0 & 150.0 & 40.0 & 60.1 & 0.0 & 0.0 & 0.1 & 0.10 & 87.7 \\
\hline 4 & 125.0 & 70.0 & 60.0 & 125 & 70.0 & 60.1 & 0.0 & 0.0 & 0.1 & 0.10 & 55.9 \\
\hline 5 & 100.0 & 100.0 & 60.0 & 100.0 & 100.0 & 60.1 & 0.0 & 0.0 & 0.1 & 0.10 & 40.0 \\
\hline 6 & 75.0 & 130.0 & 60.0 & 75.1 & 130.1 & 60.1 & 0.1 & 0.1 & 0.1 & 0.17 & 55.9 \\
\hline 7 & 50.0 & 160.0 & 60.0 & 50.1 & 160.0 & 60.1 & 0.1 & 0.0 & 0.1 & 0.14 & 87.7 \\
\hline 8 & 100.0 & 130.0 & 75.0 & 100.0 & 130.1 & 75.0 & 0.0 & 0.1 & 0.0 & 0.10 & 39.1 \\
\hline 9 & 50.0 & 40.0 & 90.0 & 50.0 & 40.0 & 90.0 & 0.0 & 0.0 & 0.0 & 0.00 & 78.7 \\
\hline 10 & 150.0 & 100.0 & 90.0 & 150.1 & 100.0 & 90.0 & 0.1 & 0.0 & 0.0 & 0.10 & 51.0 \\
\hline 11 & 125.0 & 130.0 & 90.0 & 125.1 & 130.1 & 90.0 & 0.1 & 0.1 & 0.0 & 0.14 & 40.3 \\
\hline 12 & 25.0 & 70.0 & 105.0 & 25.0 & 70.1 & 105.0 & 0.0 & 0.1 & 0.0 & 0.10 & 80.9 \\
\hline 13 & 175.0 & 130.0 & 120.0 & 175.0 & 130.1 & 120.0 & 0.0 & 0.1 & 0.0 & 0.10 & 83.2 \\
\hline 14 & 150.0 & 160.0 & 120.0 & 150.0 & 160.0 & 120.0 & 0.0 & 0.0 & 0.0 & 0.00 & 80.6 \\
\hline 15 & 100.0 & 40.0 & 135.0 & 100.1 & 40.1 & 134.9 & 0.1 & 0.1 & 0.1 & 0.17 & 69.5 \\
\hline 16 & 75.0 & 70.0 & 135.0 & 75.0 & 70.1 & 134.9 & 0.0 & 0.1 & 0.1 & 0.14 & 52.4 \\
\hline 17 & 50.0 & 100.0 & 135.0 & 50.0 & 100.0 & 134.9 & 0.0 & 0.0 & 0.1 & 0.10 & 61.0 \\
\hline 18 & 25.0 & 130.0 & 135.0 & 25.0 & 130.1 & 134.9 & 0.0 & 0.1 & 0.1 & 0.14 & 88.0 \\
\hline 19 & 175.0 & 70.0 & 150.0 & 175.0 & 70.2 & 149.8 & 0.0 & 0.2 & 0.2 & 0.28 & 95.0 \\
\hline 20 & 125.0 & 100.0 & 150.0 & 125.1 & 100.2 & 149.8 & 0.1 & 0.2 & 0.2 & 0.30 & 55.9 \\
\hline 21 & 100.0 & 160.0 & 150.0 & 100.1 & 160.1 & 149.8 & 0.1 & 0.1 & 0.2 & 0.24 & 78.1 \\
\hline
\end{tabular}

The right column delivers the distance to the position 100/100/100 (Leksell coordinate system). 


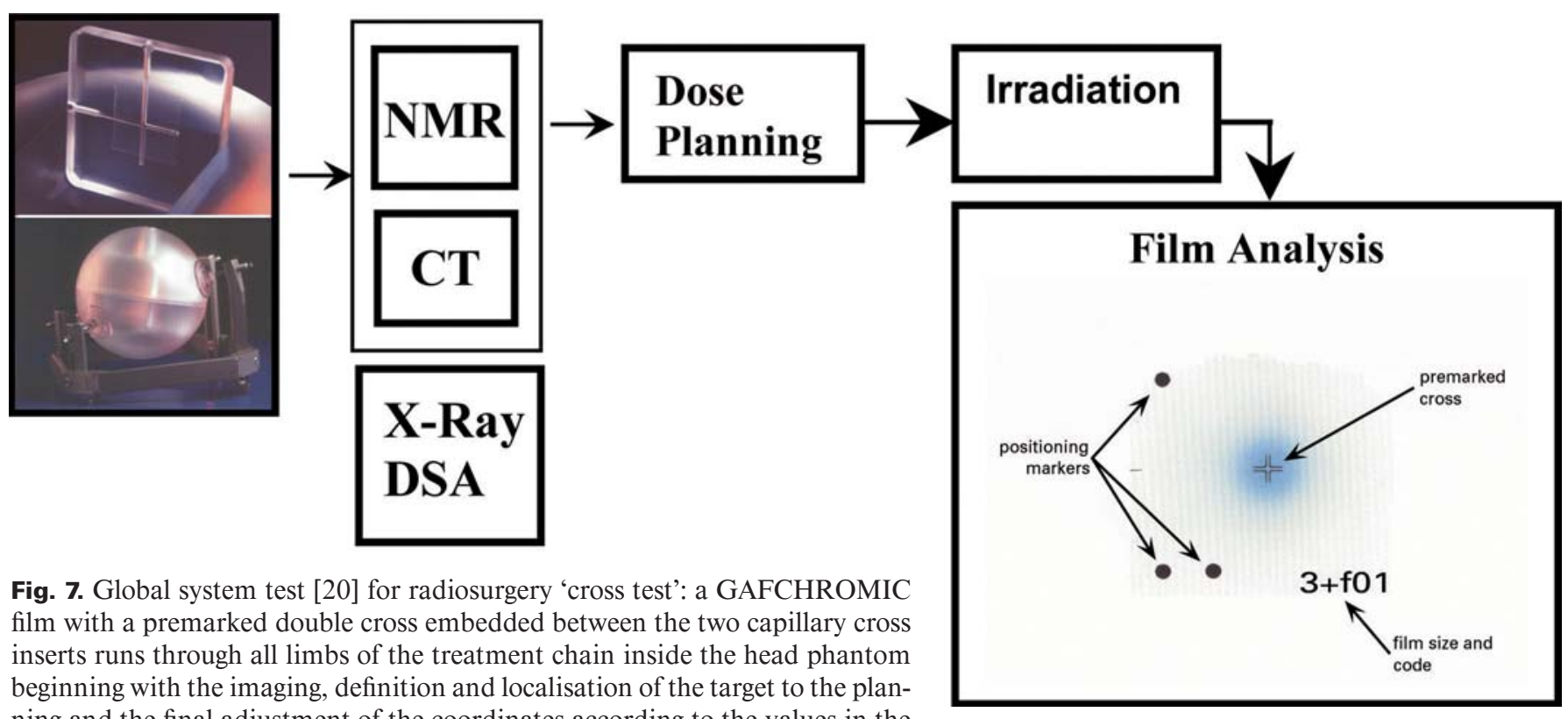
ning and the final adjustment of the coordinates according to the values in the protocol. The deviation of the coloured spot on the film to the middle of the marked double cross is evaluated. In addition to that, the imaging devices (CT, MRI) could also be checked against a reference image (X-ray anterior posterior/lateral direction).
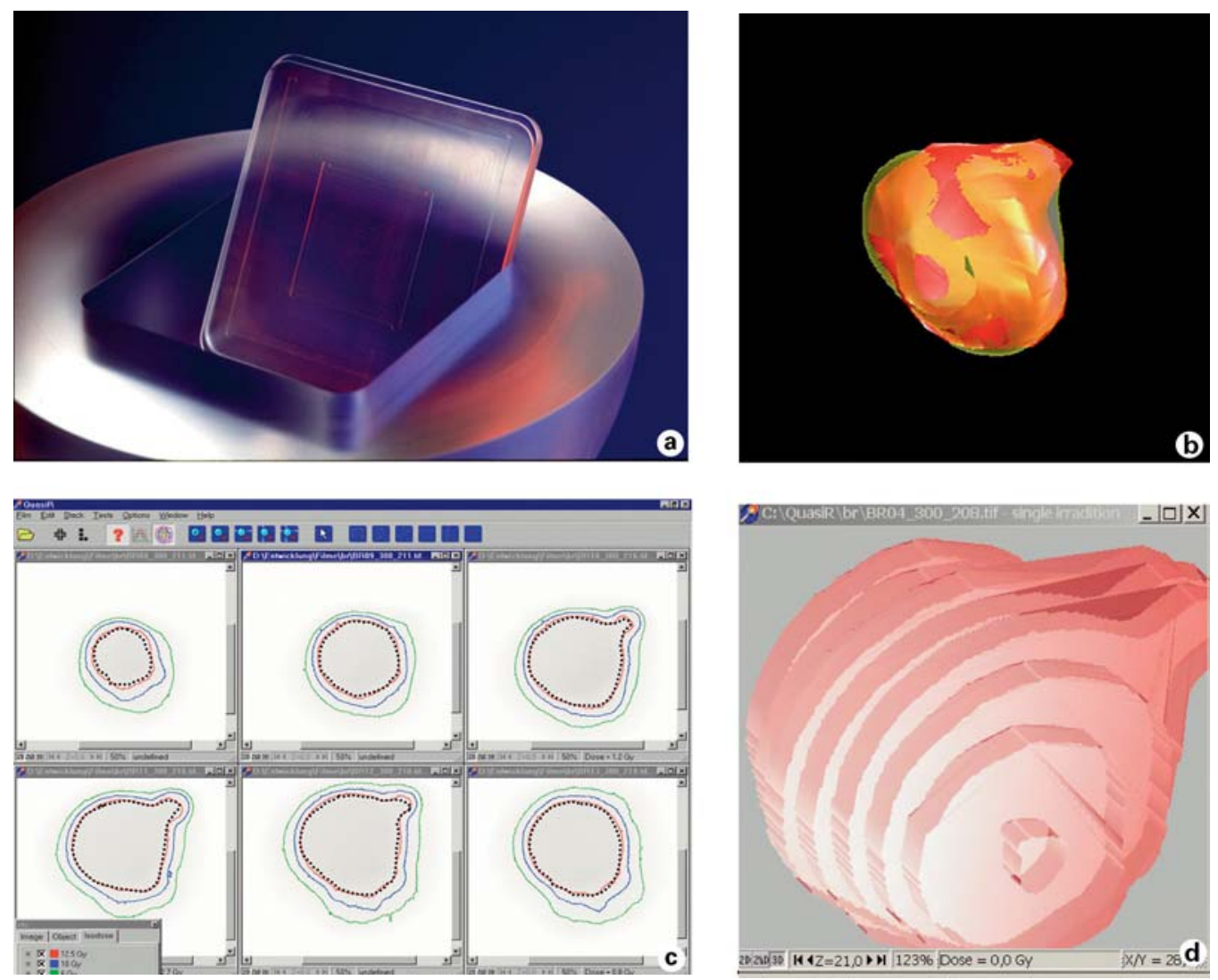

Fig. 8. Verification of a calculated 3-dimensional dose distribution for an acoustic neurinoma: the GAFCHROMIC films are embedded between the slabs and inserted into the phantom (a) which is adjusted according to the data in the protocol. The acoustic neuri- noma was planned (b) and the films are analysed. The screenshot shows dose distributions on the basis of absolute dose measurements (c) and the representation of the corresponding 3-dimensional dose plot $(\mathbf{d})$. 
Table 3. Results of 170 consecutive system tests, based on MR target point localisation

a Acquisition plan

\begin{tabular}{lccc}
\hline Deviation & All & Axial & Coronal \\
\hline Mean, mm & 0.48 & 0.42 & 0.60 \\
Sigma, mm & 0.23 & 0.24 & 0.15 \\
$\mathrm{n}$ & 170 & 116 & 54 \\
Min, mm & 0.10 & 0.10 & 0.32 \\
Max, mm & 1.12 & 1.12 & 0.92 \\
Median, mm & 0.44 & 0.32 & 0.63 \\
\hline
\end{tabular}

b MRI sequence

\begin{tabular}{lccc}
\hline Deviation & All & SE & $\begin{array}{l}\text { 3-dimensional } \\
\text { mprage }\end{array}$ \\
\hline Mean, mm & 0.48 & 0.66 & 0.40 \\
Sigma, mm & 0.23 & 0.24 & 0.19 \\
$\mathrm{n}$ & 170 & 50 & 120 \\
Min, mm & 0.10 & 0.14 & 0.10 \\
Max, mm & 1.12 & 1.12 & 0.73 \\
Median, mm & 0.44 & 0.71 & 0.36 \\
\hline
\end{tabular}

Verification of Calculated Dose Distributions

A stack of embedded films (fig. 8a) is analysed. The resulting measured dose distributions (fig. 8c, d) can be compared to the calculated ones (fig. 8b) [12, 13]. Several slab thicknesses can be chosen. The film thickness of $0.3 \mathrm{~mm}$ is the limiting parameter of the resolution orthogonal to the film plane. Several spacer plates $(1.5,3.0$ and $6 \mathrm{~mm}$ ) allow any composition of stacks. Furthermore, different materials and detectors can be combined.

\section{Conclusion}

Given the high mechanical accuracy and long-term stability of the Leksell Gamma Knife, which is $0.20 \pm$ $0.13 \mathrm{~mm}$, the most sensitive technical factor having an influence on the overall precision of radiosurgery is the MRI. Besides QA phantoms and analysis software developed in-house, which also fulfill the demands of the DIN standard, the use of commercially available tools, manufactured and distributed by the PTGR GmbH, Tübingen, Germany, facilitates the QA according to the DIN 68751 and significantly speeds up the analysis.

\section{References}

1 Mack A, Czempiel H, Kreiner HJ, Dürr G, Wowra B: Quality assurance in stereotactic space, a system test for verifying the accuracy of aim in radiosurgery. Med Phys 2002;29: 561-568.

2 Stuecklschweiger GF, Feichtinger K: A quality assurance program in stereotactic radio-surgery using the Gamma Knife unit. Strahlenther Onkol 1998;174(suppl 2):43-46.

3 Mack A, Scheib S, Major J, Gianolini S, Pazmandi G, Feist H, Czempiel H, Kreiner HJ: Precision dosimetry for narrow photon beams used in radiosurgery - Determination of Gamma Knife output factors. Med Phys 2002;29:2080-2089.

4 Mack A, Mack G, Weltz D, Scheib S, Böttcher $\mathrm{H}$, Seifert V: High precision film dosimetry with GAFCHROMIC ${ }^{\circledR}$ films for quality assurance when using small fields. Med Phys 2003; 30:2399-2409.

5 Somigliana A, Cattaneo GM, Fiorino C, Borelli S, del Vecchio A, Zonca G, Pignoli E, Loi G, Calandrino R, Marchesini R: Dosimetry of Gamma Knife and linac-based radiosurgery using radiochromic and diode detectors. Phys Med Biol 1999;44:887-897.

6 Rosenzweig DP, Schell MC, Numaguchi Y: Quality assurance in linac-based stereotactic radiosurgery and radiotherapy. Med Dosim 1998;23:147-151.
7 Meeks SL, Bova FJ, Buatti JM: Analytic characterization of linear accelerator radiosurgery dose distributions for fast optimisation. Phys Med Biol 1999;44:2777-2787.

8 Schad LR, Ehricke HH, Wowra B: Correction of spatial distortion in magnetic resonance angiography for radiosurgical treatment planning of cerebral arterio-venous malformations. Magn Reson Imaging 1992;10:609-621.

9 Mack A, Mack G, Weltz D, Hönes A, Jess A, Wowra B, Czempiel H, Heck B, Kreiner H J, Seifert V, Böttcher H: Qualitätssicherung im stereotaktischen Raum. Bestimmung der Genauigkeit von Ort und Dosis bei Einzeit-Bestrahlungen. Strahlenther Onkol 2003;179: 760-766.

10 Ertl A, Saringer W, Heimberger K: Quality assurance for the Leksell gamma unit: Considering magnetic resonance image-distortion and delineation failure in the targeting of the internal auditory canal. Med Phys 1999;26:166170 .
11 Tsai JS: Analyses of multi-irradiation film for system alignments in stereotactic radiotherapy (SRT) and radiosurgery (SRS). Phys Med Biol 1996;41:1597-1620.

12 Mack A, Weltz D, Czempiel H, Heck B, Kreiner H J, Wolff R, Mack G: Experimentally determined three-dimensional dose distributions of small, complex targets. J Neurosurg 2002; 97(suppl 5):551-555.

13 Mack A, Mack G, Weltz D, Czempiel H, Kreiner HJ: Verification of dose plans using film dosimetry for quality assurance, in Kondziolka D (ed): Radiosurgery. Basel, Karger, 2002, vol 4, pp 213-227.

14 Robar JL, Clark, BG: The use of radiographic film for linear accelerator stereotactic radiosurgical dosimetry. Med Phys 1999;26:21442150 .

15 Robar JL, Clark, BG: A practical technique for verification of three-dimensional con-formal dose distributions in stereotactic radiosurgery. Med Phys 2000;27:978-987.

16 DIN 6875-1, Ausgabe 2002-01 (Norm-Entwurf), spezielle Bestrahlungseinrichtungen. 1. Perkutane stereotaktische Bestrahlung; Kennmerkmale und besondere Prüfmethoden. Normenausschuss Radiologie. Berlin, Beuth, 2003. 